\title{
Commentary \\ N-GAL: Diagnosing AKI as soon as possible
}

Claudio Ronco

Department of Nephrology, St Bortolo Hospital, Vicenza, Italy

Corresponding author: Claudio Ronco, cronco@goldnet.it

Published: 6 November 2007

Critical Care 2007, 11:173 (doi:10.1186/cc6162)

This article is online at http://ccforum.com/content/11/6/173

(C) 2007 BioMed Central Ltd

See related research by Zappitelli et al., http://ccforum.com/content/11/5/R84

\begin{abstract}
Early diagnosis of acute kidney injury (AKI) is often problematic, due to the lack of suitable early biomarkers of renal damage and kidney function. Neutrophil gelatinase-associated lipocalin (NGAL) as an early marker of AKI partially overcomes such limitations and seems to demonstrate that diagnosing AKI in its early stages is possible and useful. Using genomic and protein microarray technology, a series of molecules have been identified as potential markers for AKI; among them NGAL has been demonstrated to rise significantly in patients with AKI but not in the corresponding controls. Furthermore, this rise in NGAL occurs in various studies at 24 to 48 hours before the rise in creatinine is observed. NGAL both in urine and plasma is an excellent early marker of AKI with an area under the receiver operator characteristic curve (AUC) in the range of 0.9 . The study of Zappitelli et al. in critically ill children combines for the first time the new RIFLE classification (Risk, Injury, Failure, Loss, End-stage renal disease) of AKI with the validation of NGAL as an early marker of kidney injury. This innovative approach brings a new hope for a timely diagnosis of $\mathrm{AKI}$ and thus a timely institution of measures for prevention and protection.
\end{abstract}

The issue of the early diagnosis of acute kidney injury (AKI) has been debated for years. Partially this has been due to the lack of a suitable and consistent definition. Other limitations are the paucity of available experimental models of $\mathrm{AKI}$ and the inadequate capability of selected marker molecules to detect the impairment of kidney function in real time. The article by Zappitelli et al. on neutrophil gelatinase-associated lipocalin (NGAL) as an early marker of acute kidney injury has partially overcome the above mentioned limitations and seems to demonstrate that diagnosing $\mathrm{AKI}$ in its early stages is possible and useful [1].

AKI prevention and therapy has as of yet been rather unsuccessful and unsatisfactory. The problem may lie in the inadequacy of the renal replacement therapies that we have applied so far; however, this is questionable and it only applies to the late stages of $\mathrm{AKI}$, when the organ function has been lost and replacement by artificial organ support is required. There are many contributions showing that technology has evolved in parallel with the worsening of the clinical conditions of the patients being treated, and it is because of this that the mortality in this condition has not changed over the years $[2,3]$. This myth is finally undergoing scientific scrutiny and the reality is emerging. We are now realizing that for a number of non-complicated AKI cases, mortality can be significantly reduced especially if an adequate renal replacement therapy is provided [4]. Nevertheless, high mortality rates still pertain to complicated cases associated to multiple organ failure or septic syndromes [5].

The story of early diagnosis is different. Only recently have we discovered that most of the preventive measures for AKI which are efficacious in the experimental settings do not show comparable positive results in the clinical setting [6]. This can be explained by the inability to identify the time of injury in the clinical setting. In the experimental models we apply the renal insult at a known time and thus we are able to apply the prevention or protection protocol timely and effectively. In the real clinical presentation it is only seldom and in specific cases (for example, elective cardiac surgery) where we can capture the exact moment of kidney insult and put in place counter measures. In order to make a solid diagnosis of $\mathrm{AKI}$ in its early phases we have been missing adequate classification and staging criteria until only a few years ago. In 2002, during the second Acute Dialysis Quality Initiative (ADQI) Consensus Conference held in Vicenza, a new classification of AKI called RIFLE (Risk, Injury, Failure, Loss, End-stage renal disease) was proposed based on the level of serum creatinine rise or urine output reduction [7]. The RIFLE classification has been validated now in many papers and it is the most current and accurate tool to define the level of $\mathrm{AKI}$ and the level of associated risk of mortality [8].

$\mathrm{ADQI}=$ Acute Dialysis Quality Initiative; $\mathrm{AKI}=$ acute kidney injury; $\mathrm{AUC}=$ area under the receiver operator characteristic curve; $\mathrm{HF}=$ heart failure; $\mathrm{NGAL}=$ Neutrophil gelatinase-associated lipocalin; RIFLE = Risk, Injury, Failure, Loss, End-stage renal disease. 
Now that we have RIFLE we may speculate that AKI can be diagnosed much earlier than in the past identifying a specific category such as risk, injury or failure. Such definitions suggest that in some cases, the AKI process has begun much earlier than can be detected by the rise in serum creatinine. Creatinine is a reliable marker of kidney function but its constant and slow production makes its rise small and late in the case of an acute injury. There remains a need for a much earlier marker in order to diagnose $\mathrm{AKI}$ as soon as possible.

Using genomic and protein microarray technology, a series of molecules have been identified as potential markers for AKI; among them NGAL which is a $25 \mathrm{kDa}$ protein, generally expressed in low concentrations, and is greatly increased in the case of epithelial damage $[1,9,10]$. In several papers NGAL has been demonstrated to rise significantly in patients with AKI but not in the corresponding controls [11-13]. Furthermore, this rise in NGAL occurs in various studies at 24 to 48 hours before the rise in creatinine is observed. NGAL both in urine and plasma is an excellent early marker of AKI with an area under the receiver operator characteristic curve (AUC) in the range of 0.9. The molecule still requires a complete evaluation in different clinical settings but the promise is both fascinating and scientifically sound.

Today, there are many studies ongoing to elucidate the nature of the association between NGAL and AKI in the critical care settings. The same holds true in the setting of different types of cardiac surgery, contrast induced nephropathy, worsening renal function in heart failure (HF) and sepsis patients.

The study of Zappitelli et al. in critically ill children combines for the first time the new RIFLE classification of AKI with the validation of NGAL as an early marker of kidney injury. This innovative approach brings a new hope for a timely diagnosis of $\mathrm{AKI}$ and thus a timely institution of measures for prevention and protection. Looking to the natural evolution of AKI, we can identify different milestones along the timeline of the syndrome. The injury begins inducing molecular modifications that subsequently evolve into cellular damage. The cells start to produce biomarkers of injury and only subsequently does the clinical picture of the syndrome develop with the typical sign and symptoms. Therefore we could imagine that the molecular and cellular clocks always anticipate the clinical clock, which is always late. The biological clock of biomarkers displays an intermediate time in this progression but it most certainly is reflective of an earlier stage when compared to the clinical clock.

We need biomarkers that are sensitive (early appearance) and specific (typical of organ injury). They must be easy to detect and measure (possibly at bedside); they must correlate with severity (offering accurate prognosis), quantitatively describing the level of injury even in the absence of typical clinical signs. Finally they must be adequate to indicate treatment initiation (theranostics) enabling future studies to compare efficiency and efficacy of therapeutic measures and techniques.

Thus, in the timeline of the AKI syndrome early biomarkers represent a unique possibility for a timely diagnosis and intervention to protect the kidney from further insults and to prevent the tissue damage from the existing risk factors. If we wait for the clinical clock to activate the alarm we will always be late. We need to diagnose AKI and treat it as soon as possible.

\section{Competing interests}

$\mathrm{CR}$ received a speaker fee from BIOSITE at the meeting of ISM in Brazil on May 2007.

\section{References}

1. Zappitelli M, Washburn KK, Arikan AA, Loftis L, Ma Q, Devarajan $\mathrm{P}$, Parikh CR, Goldstein SL: Urine neutrophil gelatinase-associated lipocalin is an early marker of acute kidney injury in critically ill children: a prospective cohort study. Crit Care 2007, 11: R84

2. Ronco C: Continuous dialysis is superior to intermittent dialysis in acute kidney injury of the critically ill patient. Nat Clin Pract Nephrol 2007, 3:118-9.

3. Ronco C, Ricci Z, Bellomo R, Baldwin I, Kellum J: Management of fluid balance in CRRT: a technical approach. Int $J$ Artif Organs 2005, 28:765-76.

4. Ronco C, Bellomo R, Homel P, Brendolan A, Dan M, Piccinni P, La Greca G: Effects of different doses in continuous venovenous haemofiltration on outcomes of acute renal failure: a prospective randomised trial. Lancet 2000; 356:26-30.

5. Chertow GM, Burcick E, Honour M, Bonventre JV, Bates DW: Acute kidney injury, mortality, length of stay, and costs in hospitalized patients. J Am Soc Nephrol 2005, 16:3365-3370.

6. Ronco C, Bellomo R: Prevention of acute renal failure in the critically ill. Nephron Clin Pract 2003, 1:C13-20.

7. Bellomo R, Ronco C, Kellum JA, Mehta RL, Palevsky P: The ADQI workgroup: Acute renal failure - definition, outcome measures, animal models, fluid therapy and information technology needs: the Second International Consensus Conference of the Acute Dialysis Quality Initiative (ADQI) Group. Crit Care 2004, 8:R204-212.

8. Uchino S, Bellomo R, Goldsmith D, Bates S, Ronco C: An assessment of the RIFLE criteria for acute renal failure in hospitalized patients. Crit Care Med 2006, 34:1913-1917.

9. Schmidt-Ott KM, Mori K, Kalandadze A, Li JY, Paragas N, Nicholas T, Devarajan P, Barasch J: Neutrophil gelatinase-associated lipocalin-mediated iron traffic in kidney epithelia. Curr Opin Nephrol Hypertens 2006, 15:442-449.

10. Cowland JB, Borregaard N: Molecular characterization and pattern of tissue expression of the gene for neutrophil gelatinaseassociated lipocalin from humans. Genomics 1997, 45: 17-23,

11. Mishra J, Dent C, Tarabishi R, Mitsnefes MM, Ma Q, Kelly C, Ruff SM, Zahedi K, Shao M, Bean J, et al.: Neutrophil gelatinaseassociated lipocalin (NGAL) as a biomarker for acute renal injury after cardiac surgery. Lancet 2005, 365:12311238.

12. Wagener G, Jan M, Kim M, Mori K, Barasch JM, Sladen RN, Lee HT: Association between increases in urinary neutrophil gelatinase-associated lipocalin and acute renal dysfunction after adult cardiac surgery. Anesthesiology 2006, 105:485491.

13. Devarajan P: Emerging biomarkers of acute kidney injury. Contrib Nephrol 2007, 156:203-212. 